\title{
Online team work in space science and astronomy at the Open University
}

\author{
M.H.Jones, S.M.Chyriwsky, J.Croston, U.Kolb, S.P.Schwenzer, S.Urquhart \\ Faculty of Science, Technology, Engineering and Mathematics \\ The Open University \\ Milton Keynes, U.K. \\ m.h.jones@open.ac.uk
}

\begin{abstract}
The UK Open University (UKOU) operates a distance-learning model supporting students who are geographically dispersed and studying part-time. Development of team-working skills is particularly challenging within such a model, but is considered a necessary part of the higher education experience of space scientists and astronomers. We report on three team-working projects in space science and astronomy that we run at the UKOU at advanced undergraduate and taught postgraduate levels. The projects are: (i) an investigation of quasar spectra using data from the Sloan Digital Sky Survey (SDSS), (ii) characterisation of variable star light curves using observations from a robotic telescope, and (iii) a Mars rover mission simulation. The robotic telescope and the Mars rover simulation are part of The Open University's award-winning OpenSTEM Labs. All three projects involve teams of students working remotely from each other and communicating through asynchronous and synchronous (shared audio and whiteboard) methods. The projects are somewhat open-ended and require teams to make collective decisions about their actions. We describe how these projects are being studied in order to better understand the student experience of on-line team-working.
\end{abstract}

Keywords - skills development; team-working; remote experimentation.

\section{INTRODUCTION}

The Open University in the United Kingdom, (UKOU) is a distance-learning higher education institution (HEI) providing undergraduate and postgraduate qualifications in a range of curriculum areas. Astrophysics is offered at advanced undergraduate level, and space science is part of a taught postgraduate program, together attracting about 120 and 50 students per annum respectively.

A skill that is important in astrophysics and space science is that of being able to work effectively with others to conduct a scientific investigation. In contrast to students at conventional HEIs, students at the UKOU have very little opportunity to meet face-to-face, and in the subject areas discussed here, all teaching is online. Thus any student group work has to be conducted by students who have only ever met together in an online setting.

Additionally, access to experimental and observational facilities also has to be conducted online. The UKOU has made very substantial progress in opening up remote experiments to students through its OpenSTEM Labs initiative [1].
Here we briefly describe three project activities that develop team-working skills and we present some early results on student interaction and student experience.

\section{TEAM PROJECTS IN ASTROPHYSICS}

Two different team projects are available to students studying astrophysics. One is based on the use of a robotic telescope to study the light curve of a variable star [2, 3]. In the other, students use the Sloan Digital Sky Survey (SDSS) [4], to investigate the optical-UV spectra of quasars. In both cases the team size is typically 6 to 10 students, and the project runs over 9 study weeks, amounting to roughly 100 hours of study.

\section{A. The robotic telescope project}

The UKOU, through its OpenSTEM Labs, operates a robotic observatory located in Tenerife, and telescope time is allocated to student groups for this project. The aim of the project is to constrain the nature of, and determine the physical characteristics of, an unclassified or poorly-studied star that shows periodic optical variability. Up to four observing nights are available to each project group allowing the acquisition of light curves using a range of photometric filters.

\section{B. The SDSS quasar project}

The SDSS is a long-standing research project (external to the UKOU) that uses a dedicated robotic telescope to conduct large-scale imaging and spectroscopic surveys. In this project, students are tasked with creating a composite quasar spectrum using SDSS data. By combining spectra from quasars with a range of redshifts, a composite spectrum can be constructed that extends from the visible to UV wavelengths.

\section{TEAM PROJECT IN SPACE SCIENCE}

The team project for the postgraduate module in space science is a simulation of a Mars rover mission. It has a much shorter duration than the astrophysics projects described above, lasting only one week and about 16 hours of study time. The simulation is based around a Mars yard facility at the UKOU campus in Milton Keynes (UK) and a robotic rover.

The scenario that teams are presented with is that "a small scouting rover has been landed on Mars to assess whether its landing site is suitable for follow-up with a large rover that will search for evidence of past or present life". The goals of the 
mission are; to document the landscape and rock types therein, to seek evidence for water-bearing minerals in those rocks, and to assess the evidence for past or present flowing or standing water. The team is expected to produce daily reports on their findings, and is responsible for the safety of the rover such that it should remain drivable throughout and at the end of the simulation.

The operating environment is a Mars yard (with approximate dimensions $6 \mathrm{~m} \times 12 \mathrm{~m}$ ) containing rocks that have been selected to be analogues of those found on Mars. The rover used in the simulation is a small, six-wheel 'all terrain' vehicle built using off-the-shelf components

Given the complexity of the rover, the mission team is split into sub-teams with responsibility for particular aspects of the mission. Typically, about 16 students participate in each simulation exercise, with 2 or 3 students allocated to each subteam.

\section{ANALYSIS}

From an educational perspective, our online collaborative projects are of interest for several reasons. Here we highlight two of several research questions that we are investigating:

- How does the content of the student forums vary depending on the nature of the projects?

- Do students perceive that engagement in online teamwork activities is important and, if so, why?

\section{A. Forum content analysis}

We have analysed the content of the online student forums that are used to support all three types of project. Forum messages, or parts thereof, are classified as relating to one of four broad categories: (i) group building and social interaction, (ii) learning and skills development, (iii) project management, and, (iv) expression of individual feelings. We analysed three forums for each of the three projects (a total of 4,187 forum posts). The proportion of messages or distinct parts of messages in these categories is shown in Table 1.

TABLE I. THE PROPORTION OF DISTINCT PARTS OF FORUM MESSAGES, CLASSIFIED BY INTENT.

\begin{tabular}{|l|l|l|l|}
\hline & \multicolumn{1}{|c|}{ SDSS } & \multicolumn{1}{|c|}{ PIRATE $^{\mathrm{a}}$} & \multicolumn{1}{|c|}{ ROVER } \\
\hline Group building & $16.8 \%$ & $15.1 \%$ & $17.9 \%$ \\
\hline Learning & $44.8 \%$ & $37.4 \%$ & $42.6 \%$ \\
\hline Project management & $36.3 \%$ & $43.8 \%$ & $37.3 \%$ \\
\hline Feelings & $2.1 \%$ & $3.6 \%$ & $2.3 \%$ \\
\hline
\end{tabular}

There are broad similarities between the projects, with a distribution which is approximately as follows: group building $-16 \%$, learning $-40 \%$, project management $-38 \%$ and feelings $-2 \%$. Given the different nature of the rover exercise (short duration, high level of synchronous communication) to the other two projects, it is notable that the content of the forums is very similar when analysed at this level. There are also interesting differences. The greater emphasis on project management in the robotic telescope project reflects the greater degree of planning and discussion needed to run a relatively complex procedure to acquire data from a telescope. Also, the expression of individual feelings was highest for the robotic telescope project - the forum posts reveal that much of this is both positive and negative sentiments associated with the challenges of operating a real telescope where the observing conditions are variable and there are sometimes technical difficulties to be overcome.

\section{B. Interviews}

We interviewed 14 volunteer students (robotic telescope: 3, SDSS: 5 rover: 6) about their experience on these team projects. The interviews addressed sixteen aspects of the student experience on these team projects. Here we report on the responses to one question: "Do you think it is important for students to engage in this type of group activity?"

The relevance of team-working to employment in the space-sector, or more generally, is clear to most students. The majority of interviewees identified clear links to employability with comments such as "if you want to work in science now, you have to collaborate with people all the time", and, "it's a key skill that's important to employers in the real world".

About half the respondents mentioned that team-working is valuable because it provides a social environment for their learning. Some mentioned the satisfaction obtained from being part of a joint-enterprise. Others reflected on the fact that being in a group can be challenging, but that their response to that challenge had been a development in their skills: "for me it's slightly out of my comfort zone, but I got more from it than anything else".

\section{Conclusions}

Online team projects in space science and astronomy at the UKOU provide a way to give distance-learning students some experience of working together in a way that reflects the way that scientific investigations are actually carried out. There are similar patterns of forum interaction between projects, but with some notable differences. We have also found that generally, students do appreciate the importance of team-working activities both for their own learning and their employability.

\section{ACKNOWLEDGMENT}

This work is supported through eSTEeM, The Open University's centre for pedagogical research in STEM.

\section{REFERENCES}

[1] T. D. Drysdale and N. S. J. Braithwaite, "An internet of laboratory things", 4th Experiment@International Conference (exp.at'17), 2017, pp. 236-240.

[2] U. Kolb, "The PIRATE Facility: At the crossroads of research and teaching", Revista Mexicana de Astronomía y Astrofísica Conference Series, 2014, vol. 45, pp. 16-19.

[3] U. Kolb, M. Brodeur, N. Braithwaite, S. Minocha, "A robotic telescope for university-level distance teaching", Robotic Telescopes, Student Research and Education Proceedings, 2018, vol. 1, pp. 127-136.

[4] D.G. York et al., "The Sloan Digital Sky Survey: technical summary", AJ, 2000, vol. 120, pp. 1579-1587. 